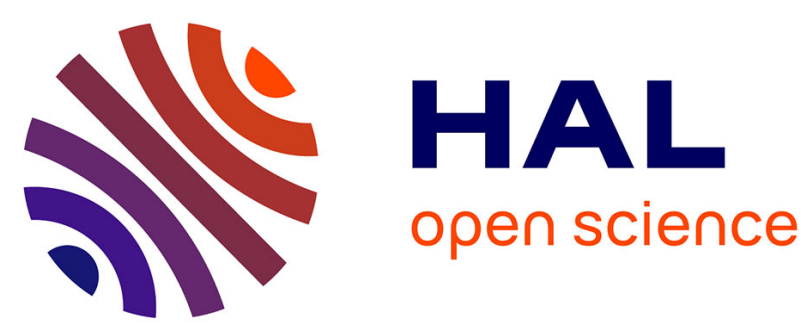

\title{
Low-Speed Cooperative Car-Following Fuzzy Controller for Cybernetic Transport Systems
}

Vicente Milanés, Mohamed Marouf, Joshué Pérez Rastelli, David Gonzalez Bautista, Fawzi Nashashibi

\section{- To cite this version:}

Vicente Milanés, Mohamed Marouf, Joshué Pérez Rastelli, David Gonzalez Bautista, Fawzi Nashashibi. Low-Speed Cooperative Car-Following Fuzzy Controller for Cybernetic Transport Systems. 2014 IEEE 17th International Conference on Intelligent Transportation Systems (ITSC), Oct 2014, Qingdao, China. 10.1109/ITSC.2014.6958009 . hal-01086877

\section{HAL Id: hal-01086877 https://hal.inria.fr/hal-01086877}

Submitted on 3 Dec 2014

HAL is a multi-disciplinary open access archive for the deposit and dissemination of scientific research documents, whether they are published or not. The documents may come from teaching and research institutions in France or abroad, or from public or private research centers.
L'archive ouverte pluridisciplinaire HAL, est destinée au dépôt et à la diffusion de documents scientifiques de niveau recherche, publiés ou non, émanant des établissements d'enseignement et de recherche français ou étrangers, des laboratoires publics ou privés. 


\title{
Low-Speed Cooperative Car-Following Fuzzy Controller for Cybernetic Transport Systems
}

\author{
Vicente Milanés, Mohamed Marouf, Joshué Pérez, David González, Fawzi Nashashibi
}

\begin{abstract}
This paper describes the development of a Cooperative Adaptive Cruise Control (CACC) for the future urban transportation system at low-speed. The control algorithm was evaluated using two Cybecars as prototype vehicles. A longitudinal response model for the vehicles was developed to design the CACC system. The control algorithm was implemented on a fuzzy logic-based controller that has been tuned to minimize a cost function in order to get a trade-off between a proper car-following gap error and the smoothness of the control signal. The controller was firstly tested in simulation using the developed model. Then, the CACC was implemented in two Cybecars to validate the controller performance in real scenarios.
\end{abstract}

\section{INTRODUCTION}

The automation of the road transportation systems is getting more and more attraction in recent years [1]. As a matter of fact, there are several actions worldwide working on this field. Among the most significant results, two of them are pretty well-known at an international level. On one hand, the University of Parma research team carried out an intercontinental autonomous trip using two electric [2]. On the other hand, Google driverless cars have carried out several public exhibitions showing autonomous capabilities. Unfortunately there are no publications about the sensor technology or control techniques that have been used for achieving those results. In spite of this significant contributions to the automation of road transport, there is still a long way to go before getting fully autonomous vehicles driving on public roads.

Although fully autonomous systems have not been yet implemented on production vehicles, there are several Advanced Driving Assistance Systems (ADAS) that are commercially available as the autonomous parking assistance systems [3] or lane keeping aid systems [4]. Among the different ADAS already in the market, the ones associated to the longitudinal control-i.e. throttle and brake pedal actionscan significantly contribute to improve traffic flow [5]. Nowadays, the most advanced deployment is the Adaptive Cruise Control (ACC) system that can be already found in mid-class vehicles [6]. This system consists on the fully automation of the longitudinal control of the vehicle combine with the use of a lidar/radar sensor located in the front of the vehicle. This sensor is in charge of detecting any preceding

Authors are with the Robotics and Intelligent Transportation Systems (RITS) Team, Inria Paris-Rocquencourt, Domaine de Voluceau, 78153 Le Chesnay, France \{vicente.milanes, mohamed.marouf, joshue.perez_rastelli, david.gonzalez-bautista, fawzi.nashashibi\} einria.fr vehicle, adapting follower's speed to keep a safe inter-vehicle distance [7].

Next steps in the research on the longitudinal control of the vehicles are focused on introducing vehicle-to-vehicle $(\mathrm{V} 2 \mathrm{~V})$ communications among vehicles, leading to the Cooperative ACC (CACC) [8]. By adding communications, inter-vehicle distances can be drastically reduced, improving traffic flow capacity [5]. Intensive studies have been carried out in the last four years on the developing of CACC systems. First implementation on real vehicles was carried out by California Partners for Advanced Transportation TecHnology (PATH) in United States using two production Infinity FX45s [9]. Field tests shown a good behavior of the car-following system. These vehicles were later used to carry out an study about the minimum time-gap that would be accepted for drivers [10]. At the same time, TNO in The Netherlands implemented a CACC controller that was later tested on six Toyota Prius III Executive [11] with good stability response. The Grand Cooperative Driving Challenge (GCDC) was a competition held in The Netherlands were nine semi-autonomous vehicles from different research institutes in Europe were equipped with CACC capabilities, competing and cooperating among them [12]. It will permit to identify the main challenges when it comes to establish V2V information exchange among vehicles with different architectures, showing that there are still some unsolved issues in CACC technology. Finally, the California PATH successfully implemented a CACC system in four production Infinity M56s. The system was tested on public roads, exhibiting a good performance [13].

Although all these developments shown good behavior, there are still some challenging scenarios that have not been further investigated. One of the most complex tasks is to control the vehicle in traffic jam situations. When the vehicle is driving at low speeds, even involved in stop\&go scenarios, it exhibits a high non-linear dynamic, making extremely difficult the control of the vehicle. As a matter of fact, most of the production ACC systems on the market are deactivated when the vehicle is involved in stop\&go scenarios or driving at low speeds. Some results have been achieved using ACC system for developing low-speed and stop\&go control algorithms [14], but it has not been yet implemented in CACC algorithms.

This paper presents the design, development and implementation of a CACC system at low speeds using a Cybercar. To this end, a prototype vehicle model was designed for analyzing speed changes vehicle response. Then, a fuzzy controller was developed to control the vehicle at low speeds. The fuzzy system was tuned by minimizing a cost func- 


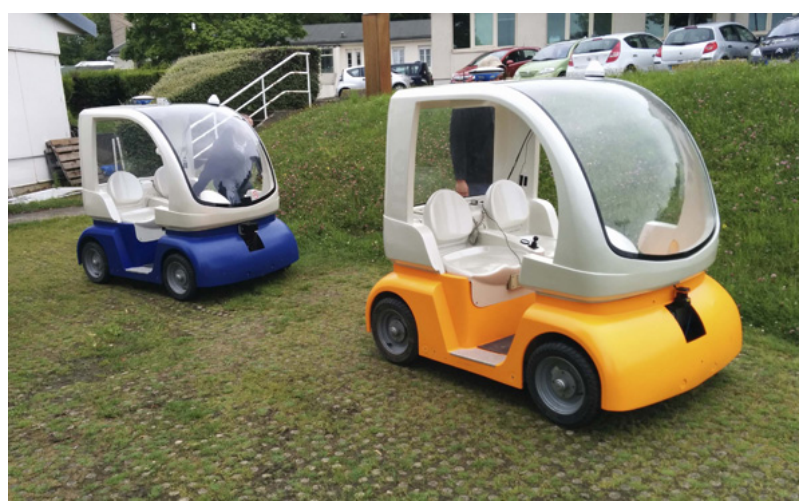

Fig. 1. Cybercar prototype vehicles

tion that considers good inter-vehicle distance tracking and smooth control actions. The controller was firstly tested in simulation; and then using two Cybercars in order to evaluate the proposed approach.

The rest of the paper is structured as follows. Section II describes the Cybercar model. The design of the fuzzy controller and how it was tuned is presented in Section III. Simulation results and on-vehicle test are included in Sections IV and V respectively. Finally, some concluding remarks and future work are given.

\section{Prototy pe Vehicle}

This section presents a brief description of the prototype vehicle that has been used-a Cybercar-and the developed vehicle model that was used to design the CACC fuzzy controller.

\section{A. Cybercar vehicle}

A Cybercar is a kind of urban transport vehicle with fully automated driving capabilities (see Fig. 1). There are several Cybercars models in function of their size, capabilities and operational environment [15], [16]. They have been conceived for urban environments, being able to reach speeds of $30 \mathrm{~km} / \mathrm{h}$ with up to four passengers. The vehicle structure is adapted from an electrical golf car frame. It has been designed with a modular architecture, being easy to drive. Each wheel motor block has its own power amplifier, driven by a micro-controller. This intelligent node consists of three linked layers. The lowest provides power to two motors. The middle layer permits sensor data acquisition and communication with the other nodes. The top layer, consisting of a Motorola MC68332 micro-controller well suited for motor control, drives the two others. Each wheel node controls the drive engine and a brake motor, with all their associated sensors (optical encoder, brake torque measurement, temperature, ...). A CAN serial bus is in charge of communicating the nodes [17], [18].

\section{B. Prototype model}

For designing the fuzzy controller, a simple second-order model of the Cybercar was developed. Desired speed is the control command that is generated by the high-level

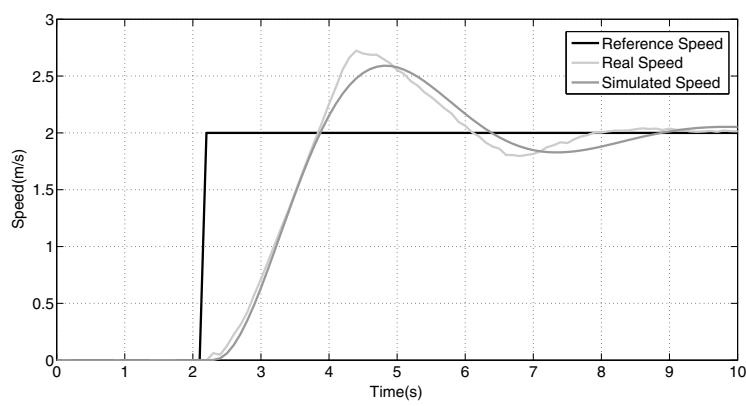

Fig. 2. Simulated and real step response of the Cybercar

controller that is sent to the low-level Proportional-IntegralDerivative (PID) controller. For modeling Cybercar vehicle, step response to different speed changes was analyzed. Figure 2 shows one of the step changes and the response of the vehicle to this change.

As it was previously stated, only low speeds were considered. One can appreciate how the Cybercar response can be approximate by a second-order function. Using the Identification Toolbox of Matlab, different transfer function models were analyzed. Figure 2 includes a comparison between the real vehicle response to a speed change and the simulated response from the second-order function. It can be clearly appreciate that the model perfectly fits (over 95\%) Cybercar's response.

The second-order function that models vehicle response to speed changes is presented in the equation below

$$
P(s)=\frac{1.0009}{0.5553 s^{2}+0.5396 s+1} e^{-0.16906 s}
$$

including a time-delay term that model the delay that is introduced by Cybercar's actuators.

\section{CONTROL ALGORITHM}

After designing a suitable model for the Cybecar response at low speeds, the CACC controller was developed. All the previous control approaches that can be found in the literature were mainly based on proportional-derivative (PD) feedback/feedforward controllers [11], [13] or Model Predictive Control (MPC) algorithms [19], [20]. In this paper, a fuzzy logic controller is used to control inter-vehicle distance.

Conventional control methods provide a good solution for developing CACC algorithm but with a high-computational cost-as occurs with MPC-or without considering human reasoning-as occurs with PD feedback/feedforward controllers. Artificial intelligence techniques can bring human driver behavior into the control loop, providing a vehicle behavior closer to what a driver can expect. Among artificial intelligence techniques, fuzzy logic is a suitable candidate because it gives a good approximation to the human reasoning and is an intuitive control technique. Clearly, a controversial point about fuzzy control systems is stability. The demonstration of string stability when it comes to design a CACC system is one of its key aspects [8]. However, this 


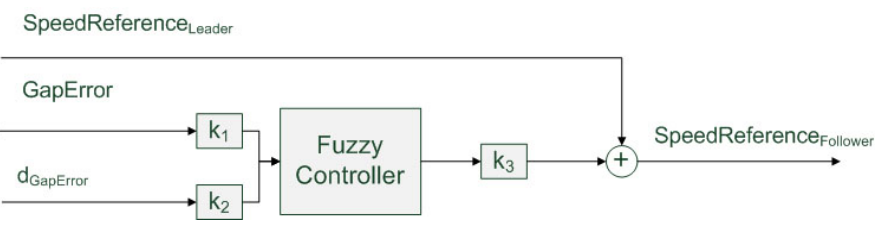

Fig. 3. Controller block diagram

string stability demonstration are based on vehicle model that can be inaccurate, compromising the stability. Usually, extensive experimental validation is often considered proof enough of stability for fuzzy systems.

The fuzzy controller developed will be in charge of generating the control commands to modify the reference follower speed, acting only over the longitudinal action of the Cybercar. Functionally, the fuzzy reasoning is done in three stages: 1) fuzzification where crisp numeric values are transformed into linguistic values; 2) inference engine where the contribution of each rule to the final output is calculated; and 3) defuzzification where the linguistic values are transformed again into crisp values.

Figure 3 shows the block diagram scheme of the control algorithm. It takes advantage of the communcation link to have access to the leading vehicle reference speed, anticipating follower's final speed. It allows to give a faster response to speed changes on the part of the leader. For a proper inter-distance tracking error, the fuzzy controller modifies the speed reference of the leading vehicle. Two variables were used as inputs for the fuzzy controller. GapError represents the error between the actual inter-vehicle distance with respect to the desired vehicle inter-distance in meters. It permits to perform a tight inter-vehicle control, following the desired reference distance; and its derivative $d_{\text {GapError }}$, permitting to respond faster to vehicle changes. The gains $k_{1}$, $k_{2}$ and $k_{3}$ will be modified in order to minimize the defined cost function.

For the desired inter-vehicle distance, the well-accepted equation $d_{d}=k_{s}+k_{g} t_{\text {gap }}$ was used; where $d_{d}$ represents the desired inter-vehicle gap, $k_{s}$ is the standstill distance and $k_{g}$ is the desired inter-vehicle gap, in seconds. This equation was already used in the GCDC competition. For our experiments, $k_{s}$ was set at $4 \mathrm{~m}$ that provide enough safety for our experimental tests and $k_{g}$ were set at $1 \mathrm{~s}$. There are recent studies were a proper inter-vehicle gap was set to $0.6 \mathrm{~s}$, according drivers acceptability [10]. However this value was defined for highway speed and it cannot be directly used for low-speed environments.

Figure 4 shows the triangular membership functions of the input variables. GapError fuzzy input variable contains seven membership functions with its seven associated linguistic labels. For the $d_{\text {GapError }}$ fuzzy input, five membership functions were defined. The output of the controller is the normalized follower reference speed change as Sugeno singletons in the interval [-1 1$]$. The algebraic product operation and the maximum union operation are applied. For the deffuzification, the crisp controller output is obtained
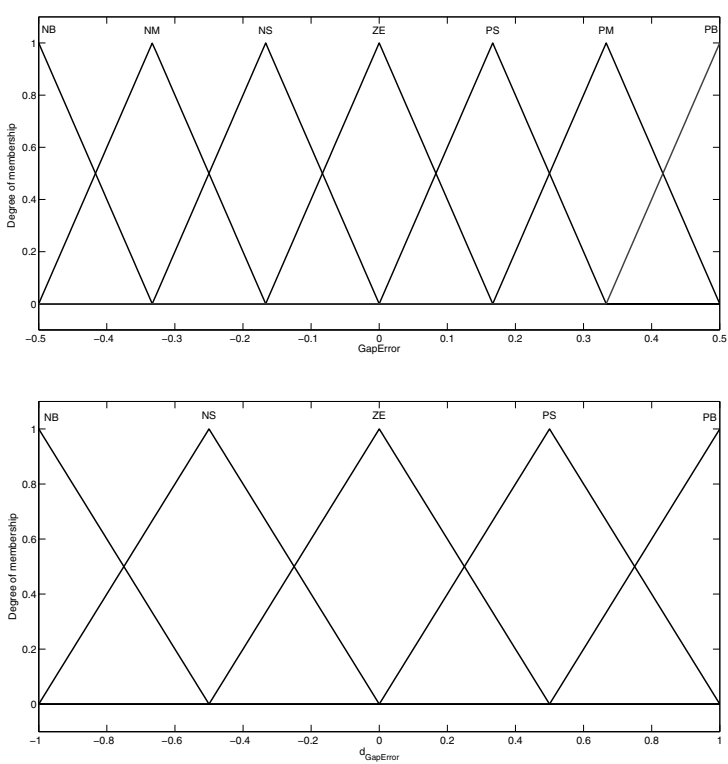

Fig. 4. Fuzzy input membership functions

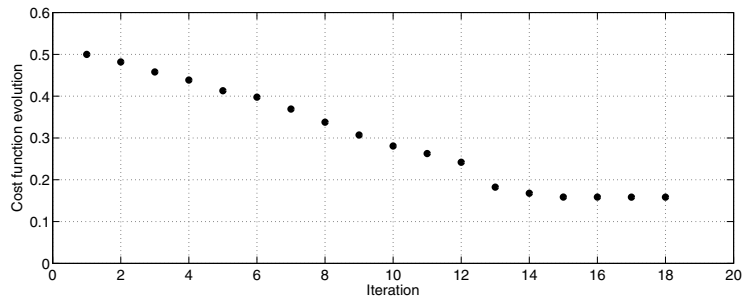

Fig. 5. Cost function evaluation for fuzzy output adjustment

using the centre-of-area (COA) method. Table I shows the controller rule base.

One of the problems associated to the used of fuzzy controllers is the absolute output generated. If the control has not been designed with enough smoothness, some control actions can cause an undesirable vehicle behavior from the passenger's standpoint. To avoid that, the fuzzy output generated is affected by a gain $\left(k_{3}\right)$. In the same way, two gains $\left(k_{1}\right.$ and $\left.k_{2}\right)$ were also adjusted for the input variables. The three gains were tuned to minimize the next cost function

$$
J=\frac{1}{T} \int_{0}^{T}\left(\left|e_{d}\right|+\left|\frac{d u_{s}(t)}{d t}\right|\right) d t
$$

where $e_{d}$ is the distance tracking error and $u_{s}$ is output of the fuzzy controller. This cost function merge the good tracking of the reference inter-vehicle distance and the smoothness of the control action. An optimization algorithm was designed to minimize $J$.

Figure 5 depicts the final evolution of the cost function values when modifying $k_{1}, k_{2}$ and $k_{3}$ following a predefined leader vehicle speed profile. It includes an acceleration and braking speed changes. Best fit was found for $k_{1}=1.2$, $k_{2}=0.9$ and $k_{3}=0.8$. 
TABLE I

CONTROLLER RULE BASE

\begin{tabular}{|c|c|c|c|c|c|c|c|}
\hline \multirow{2}{*}{$\mathbf{d}_{\text {Gap Error }}$} & \multicolumn{7}{|c|}{ GapError } \\
\cline { 2 - 8 } & NB & NM & NS & ZE & PS & PM & PB \\
\hline NB & -1 & -0.75 & -0.5 & -0.25 & 0 & 0.25 & 0.5 \\
\hline NS & -0.875 & -0.625 & -0.375 & -0.125 & 0.125 & 0.375 & 0.675 \\
\hline ZE & -0.75 & -0.5 & -0.25 & 0 & 0.25 & 0.5 & 0.75 \\
\hline PS & -0.625 & -0.375 & -0.125 & 0.125 & 0.375 & 0.625 & 0.875 \\
\hline PB & -0.5 & -0.25 & 0 & 0.25 & 0.5 & 0.75 & 1 \\
\hline
\end{tabular}
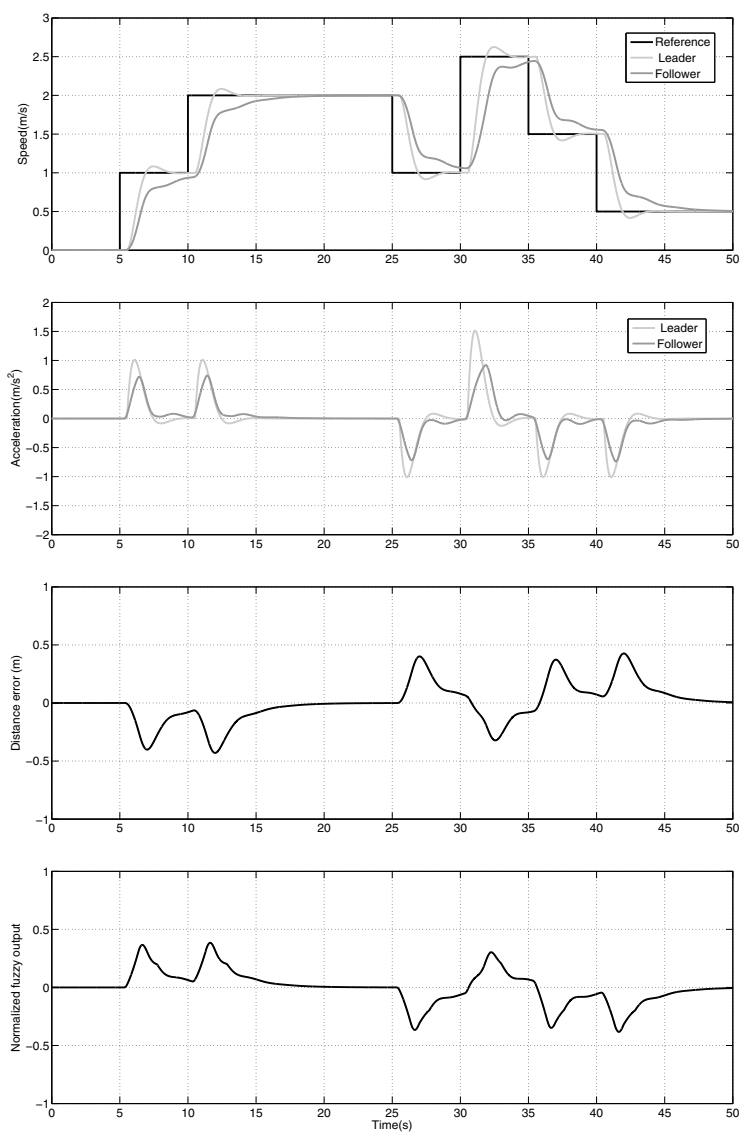

Fig. 6. Controller response to different speed changes in simulation

\section{Simulations Results}

In order to evaluate the performance of the developed fuzzy controller, some simulations were carried out. Figure 6 shows the response to different speed changes of the Cybercar using the model developed in Section II. As it was previously stated, only low speeds were considered. The top graph plots the reference speed of the leading vehicle and the response of the leader and follower to this speed profile. The next to top graph shows the Cybercars' accelerations during the simulated experiment, and the next to bottom graph shows the distance error between Cybercars. The bottom graph shows the evolution of the fuzzy controller output.

One can appreciate how the follower is responding to the leader's speed changes. Specifically, it can be noticed in the consecutive braking and accelerating maneuvers between seconds 25 and 35. Follower response is smoother, reducing significantly leader oscillations. System stability is also reflected in the accelerations graphic. Follower acceleration is considerably softer than the leader one. It increases passenger's comfort permitting a good distance tracking. The distance error when following the leader vehicle is presented in the bottom graph. In spite of the consecutive speed changes, the distance tracking error remains below $0.4 \mathrm{~m}$. It is also noticeable the smoothness in the fuzzy output value when changing vehicle's speed, according to the proper gains adjustment.

\section{REAL RESULTS}

To validate the proposed system, the fuzzy controller was implemented in a Cybecar. For the experimental test, two Cybercars were used. A speed profile was provided to the leading one, being the Cybecar equipped with the controller the trailing one. The speed profile consists on consecutive speed changes with different speed rates at low speed.

\section{A. Cybercar equipment}

To carry out the test, a V2V communication with the preceding vehicle was used to receive its reference speed; and a multilayer laser scanner was used to measure the actual distance with respect to the preceding vehicle. The study of the communication effects is out of the scope of this paper so a proper $\mathrm{V} 2 \mathrm{~V}$ communication is assumed during the tests. Experimental results validate this approach.

The laser sensor has been designed for autonomous navigation and obstacle detection of Cybercars. It proposes a strategy for grouping the laser landmarks in enhanced local maps corresponding to the roads of a Geographical Information System (GIS) layer. The laser scanner used is a laser Sick LMS-200, with 180 degree area up to 80 meters away.

The odometry information (longitudinal speed, accelerations, angle position and operating signals) comes from the low level of the vehicles, through the CAN protocol. This on-board network allows interconnection among sensors and actuators.

\section{B. Experiment}

Several trial were run where the longitudinal control of both vehicles was automated. The leader vehicle was following some speed changes with different speed rate and the follower was equipped with the CACC controller. For the lateral guidance, it was manually controlled using a joystick. The tests were carried out at Inria's facilities. 

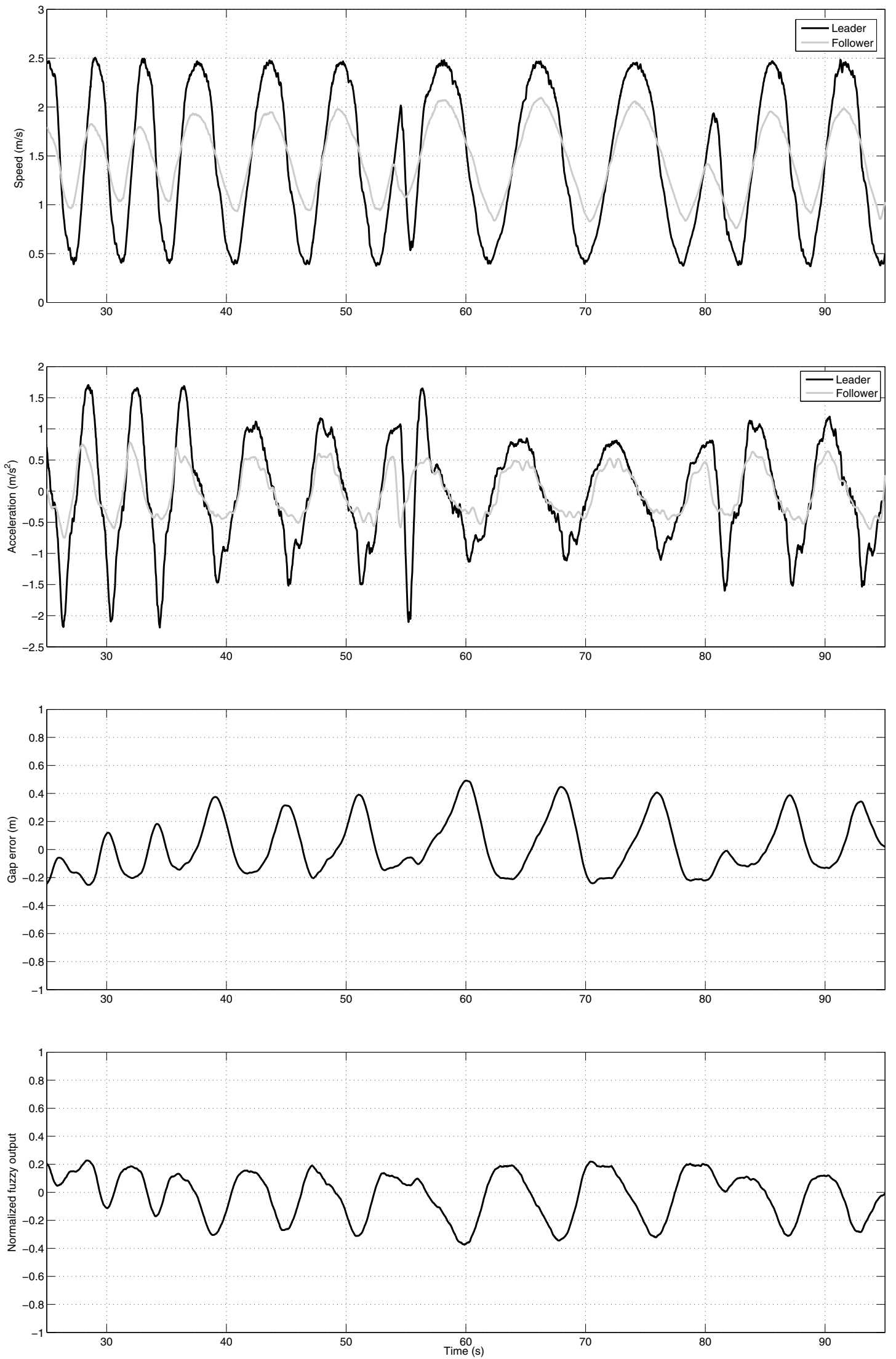

Fig. 7. Experimental results for the low speed fuzzy CACC system in the Cybercars 
Figure 7 shows the results of the experimental tests. The top graph depicts the speed of the leading (black) and of the trailing (grey) cars. The next to top graph shows the acceleration of both cars, and the next to bottom graph shows the gap error. The bottom graph shows the output of the fuzzy controller.

One can appreciate how leader speed changes are attenuated by the follower. This is also reflected on the acceleration responses, where the follower acceleration is considerably smoother than the leading one, experimentally demonstrating the stability of the designed controller. In spite of the continuous speed changes, the gap error is well-tracked during the test. Additionally, it is also noteworthy the smoothness in the fuzzy controller output response, increasing the ride-quality and verifying the proper adjustment of the gains.

\section{CONClusions AND Future Works}

The introduction of $\mathrm{V} 2 \mathrm{~V}$ communications in longitudinal control systems can significantly aid to improve traffic flow. This paper has presented a new control algorithm for CACC systems at low speeds, validating the controller using two Cybercars. For doing so, a simple model of the prototype vehicle was firstly developed. Then, a fuzzy controller was designed adjusting the input/output functions by minimizing a cost function to improve controller smoothness keeping a good inter-vehicle distance tracking. The controller was validates both in simulation and in real experiments.

The real test consisted on several speed changes on the part of the leading vehicle. The results validate the proper behavior of the proposed controller. Although string stability cannot be demonstrated using fuzzy logic, the good test response shows how fuzzy logic is a suitable candidate to be used in vehicular applications.

As future work, there are several issues that need to be further investigated. On one hand, the classical desired intervehicle distance was used. This approach achieves good results, specially in highways. However, the standstill distance can be too high for low speeds, avoiding to maximize lane capacity. For low speeds, new inter-vehicle distance generation will be studied. Additionally, the introduction of more vehicles as well as communication delay effects for longer platooning will be also analyzed in the future.

\section{ACKNOWLEDGMENTS}

The authors express their gratitude to the EU CityMobil-2 project for its support in the development of this work and the RITS team members. The work developed by Vicente Milanés is supported by a Starting position fellowship from Inria.

\section{REFERENCES}

[1] L. Li, D. Wen, N.-N. Zheng, and L.-C. Shen, "Cognitive Cars: A New Frontier for ADAS Research," IEEE Transactions on Intelligent Transportation Systems, vol. 13, no. 1, pp. 395-407, 2012.

[2] A. Broggi, P. Medici, E. Cardarelli, P. Cerri, A. Giacomazzo, and N. Finardi, "Development of the control system for the vislab intercontinental autonomous challenge," in Intelligent Transportation Systems (ITSC), 2010 13th International IEEE Conference on, 2010, pp. 635640 .
[3] G. Stanek, D. Langer, B. Muller-Bessler, and B. Huhnke, "Junior 3: A test platform for advanced driver assistance systems," in Intelligent Vehicles Symposium (IV), 2010 IEEE, 2010, pp. 143-149.

[4] B. Soualmi, C. Sentouh, J. Popieul, and S. Debernard, "Fuzzy takagisugeno lq controller for lateral control assistance of a vehicle," in Intelligent Vehicles Symposium (IV), 2012 IEEE, 2012, pp. 377-382.

[5] S. Shladover, D. Su, and X. Lu, "Impacts of cooperative adaptive cruise control on freeway traffic flow," in 91st Transportation Research Board Annual Meeting, 2012.

[6] H. Xiong and L. Boyle, "Drivers' adaptation to adaptive cruise control: Examination of automatic and manual braking," IEEE Transactions on Intelligent Transportation Systems, vol. 13, no. 3, pp. 1468-1473, 2012.

[7] J. Naranjo, C. Gonzlez, R. Garca, and T. de Pedro, "ACc+stop\&go maneuvers with throttle and brake fuzzy control," IEEE Transactions on Intelligent Transportation Systems, vol. 7, no. 2, pp. 213-225, 2006.

[8] G. Naus, R. Vugts, J. Ploeg, M. van de Molengraft, and M. Steinbuch, "String-stable cacc design and experimental validation: A frequencydomain approach," IEEE Transactions on Vehicular Technology, vol. 59, no. 9, pp. 4268-4279, 2010.

[9] F. Bu, H.-S. Tan, and J. Huang, "Design and field testing of a cooperative adaptive cruise control system," in Proc. American Control Conf. (ACC), 2010, pp. 4616-4621.

[10] J. Nowakowski, C.and OConnell, S. Shladover, and D. Cody, "Cooperative adaptive cruise control: Driver acceptance of following gap settings less than one second," in Proceedings of the Human Factors and Ergonomics Society 54th Annual Meeting. The Human Factors and Ergonomics Society, 2010.

[11] J. Ploeg, B. T. M. Scheepers, E. van Nunen, N. van de Wouw, and H. Nijmeijer, "Design and experimental evaluation of cooperative adaptive cruise control," in Proc. 14th Int Intelligent Transportation Systems (ITSC) IEEE Conf, 2011, pp. 260-265.

[12] E. van Nunen, M. Kwakkernaat, J. Ploeg, and B. Netten, "Cooperative competition for future mobility," IEEE Transactions on Intelligent Transportation Systems, vol. 13, no. 3, pp. 1018-1025, 2012.

[13] V. Milanes, S. Shladover, J. Spring, C. Nowakowski, H. Kawazoe, and M. Nakamura, "Cooperative adaptive cruise control in real traffic situations," IEEE Transactions on Intelligent Transportation Systems, vol. 15 , no. 1, pp. 296-305, 2014.

[14] V. Milanes, J. Villagra, J., J. Godoy, and C. Gonzalez, "Comparing fuzzy and intelligent pi controllers in stop-and-go manoeuvres," IEEE Transactions on Control Systems Technology, vol. 20, no. 3, pp. 770778, 2012.

[15] M. Parent, G. Gallis, A. Alessandrini, and T. Chanard, "Cybercars: Review of first projects," Inria,University of Rome and GEA- Lausanne, Tech. Rep., 2003.

[16] Y. Ming, W. Chen, Y. Ruqing, and M. Parent, "Cyberc3: Cybercar automated vehicles in china," in Transportation Research Board 85th Annual Meeting, 2006.

[17] N. Morette, C. Novales, and P. Vieyres, "Inverse versus direct kinematics model based on flatness and escape lanes to control cycab mobile robot," in Robotics and Automation, 2008. ICRA 2008. IEEE International Conference on, 2008, pp. 2240-2245.

[18] V. Milanes, J. Alonso, L. Bouraoui, and J. Ploeg, "Cooperative maneuvering in close environments among cybercars and dual-mode cars," IEEE Transactions on Intelligent Transportation Systems, vol. 12, no. 1, pp. 15-24, 2011.

[19] A. Geiger, M. Lauer, F. Moosmann, B. Ranft, H. Rapp, C. Stiller, and J. Ziegler, "Team annieway's entry to the 2011 grand cooperative driving challenge," IEEE Transactions on Intelligent Transportation Systems, vol. 13, no. 3, pp. 1008-1017, 2012.

[20] R. Kianfar, B. Augusto, A. Ebadighajari, U. Hakeem, J. Nilsson, A. Raza, R. Tabar, V. N., C. Englund, P. Falcone, S. Papanastasiou, L. Svensson, and H. Wymeersch, "Design and experimental validation of a cooperative driving system in the grand cooperative driving challenge," IEEE Transactions on Intelligent Transportation Systems, vol. 13, no. 3, pp. 994-1007, 2012. 